\title{
RELIABILITY ANALYSIS OF NETWORKS INTERCONNECTED WITH COPULAS
}

\author{
Jasper Behrensdorf ${ }^{1}$, Matteo Broggi ${ }^{1}$, and Michael Beer ${ }^{1,2,3}$ \\ ${ }^{1}$ Institute for Risk and Reliability, Leibniz University Hannover, Hannover, Germany. \\ Email: behrensdorf@irz.uni-hannover.de \\ ${ }^{2}$ Institute for Risk and Uncertainty, University of Liverpool, Liverpool, UK \\ ${ }^{3}$ International Joint Research Center for Engineering Reliability and Stochastic \\ Mechanics (ERSM), Tongji University, Shanghai, China
}

\begin{abstract}
With the increasing size and complexity of modern infrastructure networks rises the challenge of devising efficient and accurate methods for the reliability analysis of these systems. Special care must be taken in order to include any possible interdependencies between networks and to properly treat all uncertainties. This work presents a new approach for the reliability analysis of complex interconnected networks through Monte Carlo Simulation and survival signature. Application of the survival signature is key in overcoming limitations imposed by classical analysis techniques and facilitating the inclusion of competing failure modes. The (inter)dependencies are modelled using vine copulas while the uncertainties are handled by applying probability-boxes and imprecise copulas. The proposed method is tested on a complex scenario based on the IEEE reliability test system, proving it's effectiveness and highlighting the ability to model complicated scenarios subject to a variety of dependent failure mechanisms.
\end{abstract}

\section{INTRODUCTION}

Reliability analysis of complex networks is an important task in the field of risk analysis. This importance is a result of the ever increasing size and complexity of modern critical infrastructure. At the same time, society is becoming increasingly reliant on the availability of these critical infrastructures such as water supply networks, electrical distribution networks or the internet. A breakdown of any of these systems can have a drastic impact on people's lives, as evident from the aftermath of recent natural disasters [1]. As a result, efficient and accurate methods for the reliability of these complex systems are required. However, history has shown that it is not sufficient to analyse these networks as individual units because the systems are often subject to 
complex interdependencies between one another. That is, failure in one network can potentially cascade into another network $[2,3]$. For example, failures in a power grid due to natural disasters will drastically effect the communication network which in turn will inhibit the coordination of emergency personnel [4]. Therefore, it is of paramount importance to include and accurately model these interdependencies when analysing the reliability of networks.

Behrensdorf, Brandt, Broggi, and Beer [5] presented a novel approach to the numerical reliability analysis of interdependent networks based on Monte Carlo simulation and survival signature. The survival signature has the capability to fully separate the structure of a network from its probabilistic characteristics, allowing for efficient simulation while modelling dependencies in a probabilistic way [6]. Due to these characteristics it has constantly increased in popularity since its development, with new simulations techniques based on the signature being constantly developed (see for example [7]). In the previous the modelling of interdependencies between networks was limited to simple deterministic unidirectional causal links where failure of one component would result in the immediate failure of all dependent components. However, this approach lacks flexibility and does not allow to accurately capture the complex interdependencies between real world networks. As a result, a new methodology to model these interdependencies is required. Copulas have been successfully used to model dependence in enterprise risk management, finance, insurance, and environmental studies [8-11]. Modelling dependencies with copulas is especially powerful as multivariate copulas allow to separate modelling of the marginal distributions from modelling the dependence structure [12]. Though the popularity of copulas for engineering applications has increased in the recent years [13, 14], literature is still scarce.

This work extends the previously developed method to allow for complex dependencies between nodes and networks as well as competing failure modes using multivariate copulas. This work is focused on using appropriate copulas to represent realistic dependency structures between different networks. The goal is to find a single dependency structure containing the complete dependency information. For this reason, different types of multivariate copulas such as hierarchical Archimedean copulas and vine copulas are investigated. The copula models are usually inferred from data or expert knowledge, both of which are subject to two types of uncertainty, namely aleatory and epistemic uncertainty. Aleatory uncertainty represents the natural randomness in process while epistemic uncertainty results from vagueness or lack of information [15]. Dealing with these uncertainties by imprecise reliability analysis results in bounds on the obtained survival function.

The remainder of this paper is outlined as follows. First, the basic notations and required definitions of copulas including measures of dependence is presented, followed by a discussion of copula construction methods. Then, the approach to modelling dependencies is presented. Next, the numerical method used to compute the network reliability is introduced. After discussion methods to handle uncertainties in the analysis, the proposed method is applied to a complex numerical example. Finally, the paper closes with some concluding remarks and an outlook into future works. 


\section{COPULAS}

This chapter introduces the basic theory on copulas as well as how they can be used to model dependencies in high dimensions. An overview of different parametric copula families is given. Additionally, measurements of dependence are introduced. For a comprehensive discussion of copulas, see for example [16] or [12].

Copulas (from the Latin for 'bond' or 'tie') are functions that couple multivariate distribution functions to their one-dimensional marginal distributions functions and as such allow to separate modelling of the dependence structure from modelling the univariate marginals [16]. The foundation of the theory of copulas lies in what is known as Sklar's theorem [17]. It states, that any multivariate distribution $H$ can always be separated into its marginal distributions $F_{i}$ and a copula function $C$. The theorem is valid in all dimensions $d \geq 2$.

Theorem 2.1 (Sklar's theorem) Let $H$ be an d-dimensional distribution function with margins $F_{1}, \ldots, F_{d}$. There exists an d-dimensional copula $C$ such that for all $\mathbf{x}$ in $\mathbb{R}^{d}$

$$
H(\mathbf{x})=C\left(F_{1}\left(x_{1}\right), \ldots, F_{d}\left(x_{d}\right)\right) .
$$

If the marginals $F_{1}, \ldots, F_{d}$ are continuous, then $C$ is unique; otherwise, $C$ is unique on Range $\left(F_{1}\right) \times \cdots \times$ Range $\left(F_{d}\right)$. Conversely, if $C$ is a $d$-copula and $F_{1}, \ldots, F_{d}$ are distribution functions, then the function $H$ defined by $E q .1$ is an d-dimensional distribution function with margins $F_{1}, \ldots, F_{d}$.

Probabilistically, if $C$ is a joint cumulative distribution function of a $d$-dimensional random vector on the unit cube $[0,1]^{d}$ with uniform marginals, then $C:[0,1]^{d} \rightarrow$ $[0,1]$ is a copula. It is noteworthy, that copulas are invariant under strictly increasing transformations, as stated by Theorem 2.2 [16].

Theorem 2.2 For $d \geq 2$ let $X_{1}, \ldots, X_{d}$ be random variables with continuous distribution functions $F_{1}, \ldots, F_{d}$, joint distribution function $H$ and copula $C$. Let $f_{1}, \ldots, f_{d}$ be strictly increasing functions from $\mathbb{R}$ to $\mathbb{R}$. Then $f_{1}\left(X_{1}\right), \ldots, f_{d}\left(X_{d}\right)$ are random variables with continuous distribution functions and copula $C$. Thus, $C$ is invariant under strictly increasing transformation of $X_{1}, \ldots, X_{d}$.

As such, any property of the joint distribution function that is invariant under strictly increasing transformation is in fact a property of the copula. As a result, this means, one can study dependence between random variables by studying the copula [8]. There exist multiple copula families with different dependence structures of which some of the most popular are presented in the following.

\section{The Gaussian Copula}

The $d$-dimensional Gaussian copula with positive definite correlation Matrix $\mathbf{R} \in$ $[-1,1]^{d \times d}$ is defined by

$$
C_{R}\left(u_{1}, \ldots, u_{d}\right)=\Phi_{d}\left(\Phi^{-1}\left(u_{1}\right), \ldots, \Phi^{-1}\left(u_{d}\right)\right),
$$

where $\Phi_{d}(\cdot ; \mathbf{R})$ is the $d$-variate cumulative distribution of a $\mathbb{N}_{d}(0, \mathbf{R})$ random vector and $\Phi^{-1}$ denotes the inverse of the univariate standard Gaussian cumulative distribution function [12]. 
Table 1. Most popular Archimedean copulas with generators, generator inverses, and parameter domains.

\begin{tabular}{llll}
\hline \hline Name & Generator $\varphi_{\theta}(t)$ & Generator Inverse $\varphi_{\theta}^{-1}(t)$ & Parameter \\
\hline \hline Ali-Mikhall-Haqlog $\left(\frac{1-\theta(1-t)}{t}\right)$ & $\frac{1-\theta}{\exp (t)-\theta}$ & $\theta \in[-1,1)$ \\
Clayton & $\frac{1}{\theta}\left(t^{-\theta}-1\right)$ & $(1+\theta t)^{-1 / \theta}$ & $\theta \in[-1, \infty) \backslash\{0\}$ \\
Frank & $-\log \left(\frac{\exp (-\theta t)-1}{\exp (-\theta)-1}\right)$ & $-\frac{1}{\theta} \log (1+\exp (-t)(\exp (-\theta)-1))$ & $\theta \in \mathbb{R} \backslash\{0\}$ \\
Gumbel & $(-\log (t))^{\theta}$ & $\exp \left(-t^{1 / \theta}\right)$ & $\theta \in[1, \infty)$ \\
Independence & $-\log (t)$ & $\exp (-t)$ & \\
Joe & $-\log \left(1-(1-t)^{\theta}\right)$ & $1-(1-\exp (-t))^{1 / \theta}$ & $\theta \in[1, \infty)$ \\
\hline \hline
\end{tabular}

\section{Archimedean Copulas}

Archimedean copulas are an important class of copulas. Their popularity stems from a variety of reasons: they are easily constructed, the class holds a great number of different families and the copulas posses many excellent properties [16]. Additionally, the bivariate Archimedean copulas can be used in multivariate construction methods based on pairs of bivariate copulas [12]. A $d$-dimensional copula $C_{\varphi}$ is classified as Archimedean if it admits to the representation

$$
C_{\varphi}\left(u_{1}, \ldots, u_{d}\right):=\varphi\left(\varphi^{-1}\left(u_{1}\right)+\cdots+\varphi^{-1}\left(u_{d}\right)\right) \text {, }
$$

where the function $\varphi:[0, \infty] \rightarrow[0,1]$ is called the generator of $C_{\varphi}, \varphi^{-1}$ denotes its inverse and $u_{1}, \ldots, u_{d} \in[0,1]$ [18]. Table 1 shows some of the most popular oneparameter (governing the strength of dependence) Archimedean copula families with their generators, inverses and parameter domains.

\section{Dependence}

The study of dependence among random variables requires some form of dependence measurement. Typically, 'correlation' is used to describe different forms of dependence. However, in its technical meaning as the linear correlation coefficient $\rho$ it is not 'scale-invariant' and as such does not remain unchanged under strictly increasing transformation [8]. Therefore, the more modern term 'association' is used instead of correlation. Two well known scale-invariant measures of association are the population versions of Kendall's tau and Spearman's rho. In this work, Kendall's tau is applied in all cases.

Kendall's tau is a measure of association based on concordance. A pair of random variables is concordant if 'large' values of one are associated with 'large' values of the other and the same holds for 'small' values. Formally, two observations $\left(x_{i}, y_{i}\right)$ and $\left(x_{j}, y_{j}\right)$ from a vector $(X, Y)$ are concordant if $x_{i}<x_{j}$ and $y_{i}<y_{j}$, or discordant if $x_{i}>x_{j}$ and $y_{i}>y_{j}$. Alternatively, concordance can be expressed as $\left(x_{i}-x_{j}\right)\left(y_{i}-y_{j}\right)>0$ and discordance as $\left(x_{i}-x_{j}\right)\left(y_{i}-y_{j}\right)<0$.

Let $(X, Y)$ denote a vector of continuous random variables and $\left\{\left(x_{i}, y_{i}\right), \ldots,\left(x_{n}, y_{n}\right)\right\}$ a sample of $n$ observations from said vector. With $c$ as the number of concordant pairs 


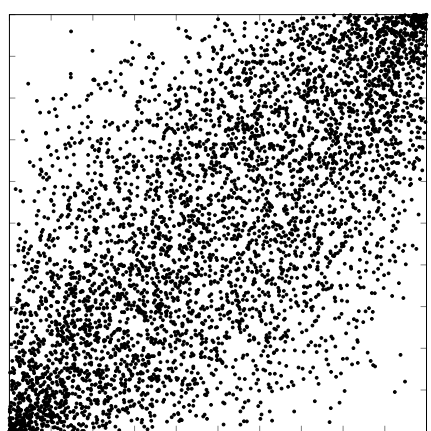

(a) Gaussian

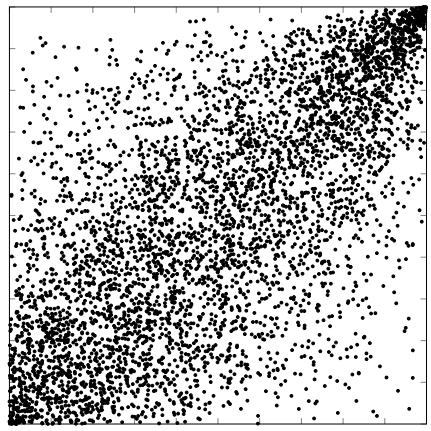

(c) Gumbel

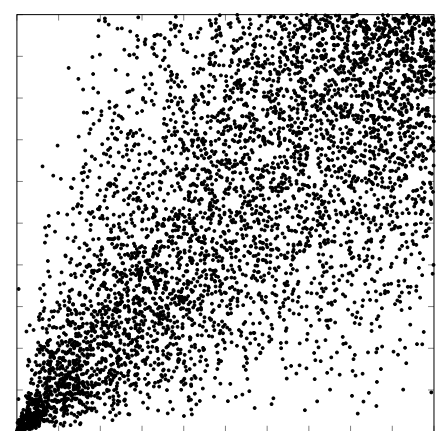

(b) Clayton

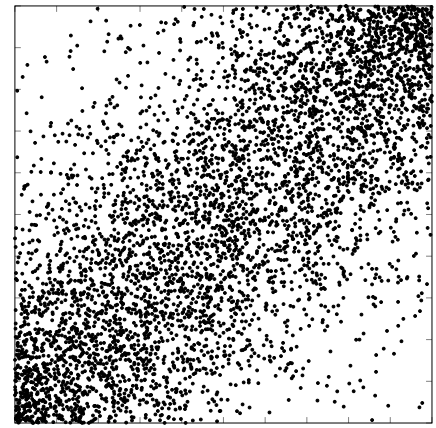

(d) Frank

Fig. 1. Samples drawn from different bivariate copulas where the parameters have been chosen so that Kendall's tau equals 0.5 .

and $d$ the number of discordant pairs among all possible $\left(\begin{array}{l}n \\ 2\end{array}\right)$ pairs of observations $\left(x_{i}, y_{i}\right)$ and $\left(x_{j}, y_{j}\right)$, Kendall's tau for the sample is defined as

$$
t=\frac{c-d}{c+d}=(c-d) /\left(\begin{array}{l}
n \\
2
\end{array}\right) \text {. }
$$

The value $t$ may also be interpreted as the probability of concordance minus the probability of discordance for a random pair of observations $\left(x_{i}, y_{i}\right)$ and $\left(x_{j}, y_{j}\right)$ chosen from the sample. In turn, this can be applied to define the population version of Kendall's tau for random variables $X$ and $Y$

$$
\tau(X, Y)=P[(X-\widetilde{X})(Y-\widetilde{Y})>0]-P[(X-\widetilde{X})(Y-\widetilde{Y})<0],
$$

where $(\widetilde{X}, \widetilde{Y})$ is an independent pair with the same distributions as $(X, Y)$ [8]. Figure 1 shows four example scatter plots of samples generated from different bivariate copulas with the respective parameters chosen such that Kendall's tau equals 0.5, highlighting the individual dependence structure.

\section{COPULA CONSTRUCTION METHODS}

Modelling dependencies inside and between networks requires a flexible dependence structure. Using one distinct copula family to sample failure times for all components 


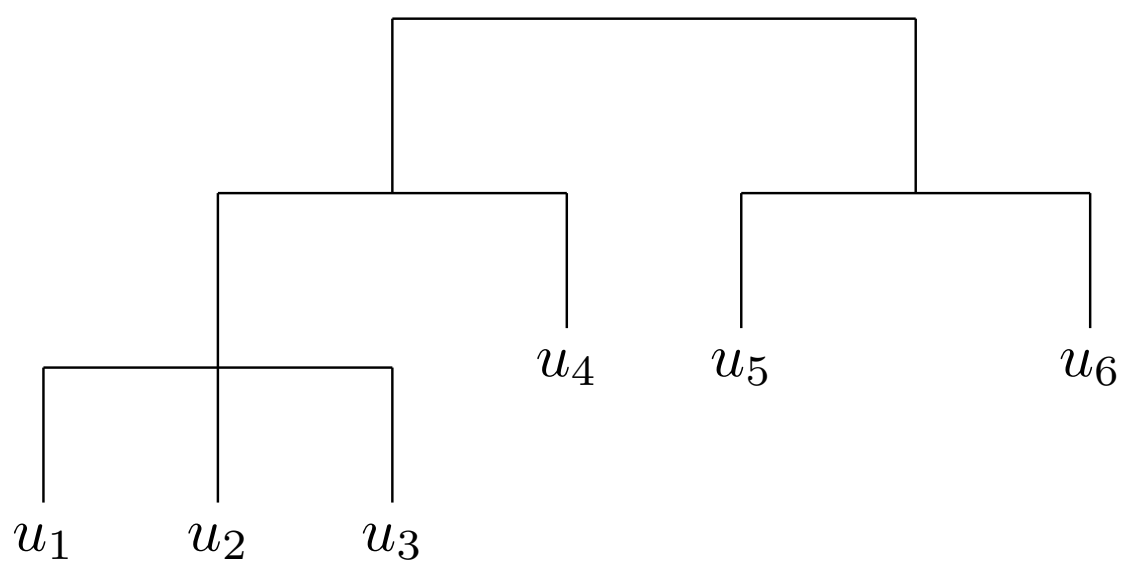

Fig. 2. Structure of a 6-dimensional hierarchical Archimedean copula

in one or multiple networks is never precise enough. Therefore, the ability to combine different copula families in one structure is of utmost importance. This section presents two copula construction methods capable of this. These methods possess different modelling capabilities and strengths. For a discussion of additional methods and further details, see [12].

\section{Hierarchical Archimedean Copulas}

Hierarchical (alternatively: nested) Archimedean copulas are a class of copulas where groups of variables are connected by Archimedean copulas and these groups themselves are then coupled with another copula from one of the Archimedean families. This nesting structure may be repeated up to an arbitrary number of nesting levels. Figure 2 shows a visual representation of a hierarchical Archimedean copula with six variables in four groups as a dendrogram. Formally, hierarchical Archimedean copulas are defined by

$$
C_{\varphi_{0}}\left(C_{\varphi_{1}}\left(u_{1,1}, \ldots, u_{1, d_{1}}\right), \ldots, C_{\varphi_{J}}\left(u_{J, 1}, \ldots, u_{J, d_{J}}\right)\right)
$$

where further nesting levels are defined recursively [18]. However, not all arbitrary combinations of $J+1$ generators lead to Eq. 6 defining a valid copula.

The dependence in every group in this structure is governed by one parameter and variables that are close to each other (e.g, in the same group) share the same dependence [12]. This reduces the modelling flexibility substantially. An implementation of hierarchical Archimedean copulas can be found in the package nacopula for the statistical programming language $\mathbf{R}$ [19].

\section{Pair Copula Construction}

The goal of pair copula constructions (PCCs) is to build high-dimensional copulas from combinations of bivariate copulas and as such use the extensive theory on bivariate copulas to overcome limitations in the available literature on multivariate copulas [18].

Consider a vector of $d$ random variables $\mathbf{X}=\left(X_{1}, \ldots, X_{d}\right)$ with joint density function denoted by $f_{1: d}\left(x_{1}, \ldots, x_{d}\right)$. The density can then be represented as a factorization of 
conditional densities:

$f_{1: d}\left(x_{1}, \ldots, x_{d}\right)=f_{1}\left(x_{1}\right) \cdot f_{2 \mid 1}\left(x_{2} \mid x_{1}\right) \cdot f_{3 \mid 2,1}\left(x_{3} \mid x_{2}, x_{1}\right) \cdots f_{d \mid 1:(d-1)}\left(x_{d} \mid x_{1}, \ldots, x_{d-1}\right)$

In the next step Sklar's theorem is applied to the conditional densities effectively splitting a multivariate density into bivariate copula densities and densities of univariate margins. Differentiating Eq. 1 with respect to a distribution with joint density $f\left(x_{1}, \ldots, x_{d}\right)$, marginals $f_{j}$ and marginal cdfs $F_{j}, j=1, \ldots, d$ leads to

$$
f_{1: d}\left(x_{1}, \ldots, x_{d}\right)=c_{1: d}\left(F_{1}\left(x_{1}\right), \ldots, F_{d}\left(x_{d}\right)\right) \cdot f_{1}\left(x_{1}\right) \cdots f_{d}\left(x_{d}\right),
$$

where $c_{1: d}(\cdot)$ is the $d$-variate copula density. The bivariate case with pair-copula density $c_{1,2}(\cdot, \cdot)$ simplifies to

$$
f_{1,2}\left(x_{1}, x_{2}\right)=c_{1,2}\left(F_{1}\left(x_{1}\right), F_{2}\left(x_{2}\right)\right) \cdot f_{1}\left(x_{1}\right) \cdot f_{2}\left(x_{2}\right),
$$

which yields

$$
f_{1 \mid 2}\left(x_{1} \mid x_{2}\right)=c_{1,2}\left(F_{1}\left(x_{1}\right), F_{2}\left(x_{2}\right)\right) \cdot f_{1}\left(x_{1}\right) .
$$

Equation 10 can be applied stepwise to Eq. 7 to fully decompose the multivariate density into bivariate copula densities and densities of univariate marginals. Note, that not all multivariate copulas can be modelled with this pair copula construction method.

\section{Vine Copulas}

Vines are a graphical representation of valid pair copula decompositions as sets of trees. Basic graph theory is used to define vines [18]. A regular vine (R-Vine) $\mathcal{V}=\left(T_{1}, \ldots, T_{d-1}\right)$ is defined as a tree sequence on $d$ elements where:

1. $T_{1}$ is a tree with Nodes $N 1=\{1, \ldots, d\}$ and edges $E_{1}$.

2. For $j \geq 2, T_{j}$ is a tree with nodes $N_{j}=E_{j-1}$ and edges $E_{j}$.

3. For $j=2, \ldots, d-1$ and $\{a, b\}$ it must hold that $|a \cap b|=1$.

The so called proximity property (3) states that, if an edge exists in $T_{j}, j \geq 2$ connecting $a$ and $b$, in turn $a$ and $b$ must share a common node in $T_{j-1}$. Figure 3 shows a regular vine representation of a 5-dimensional copula. There exist a multitude of $d$-dimensional R-vines. However, two sub-classes called C- and D-Vines are used almost exclusively. A regular vine $\mathcal{V}$ is called a $\mathrm{C}$-Vine if in each tree $T_{i}$ there is one node that holds $n \in N_{i}$ such that $\left|\left\{e \in E_{i} \mid n \in e\right\}\right| \leq d-1$. This condition states, that in each tree one node has the maximum degree (is connected to all other nodes). Alternatively, a D-Vine is characterised by each node $n \in N_{i}$ satisfying $\left|\left\{e \in E_{i} \mid n \in e\right\}\right| \leq 2$. Thus, any node may only have a maximum of two connections. Figure 4 shows the graphical structures of a five-dimensional C- and D-vine. Sampling of vine copulas is a nontrivial task. A regular vine on $n$ variables possesses $2^{n-1}$ implied sampling orders [18]. Therefore, C- and D-Vines, where sampling is easier, are applied in all examples of this work with sampling from the vines being performed by the MATLAB toolbox VineCopulaMatlab [20]. 

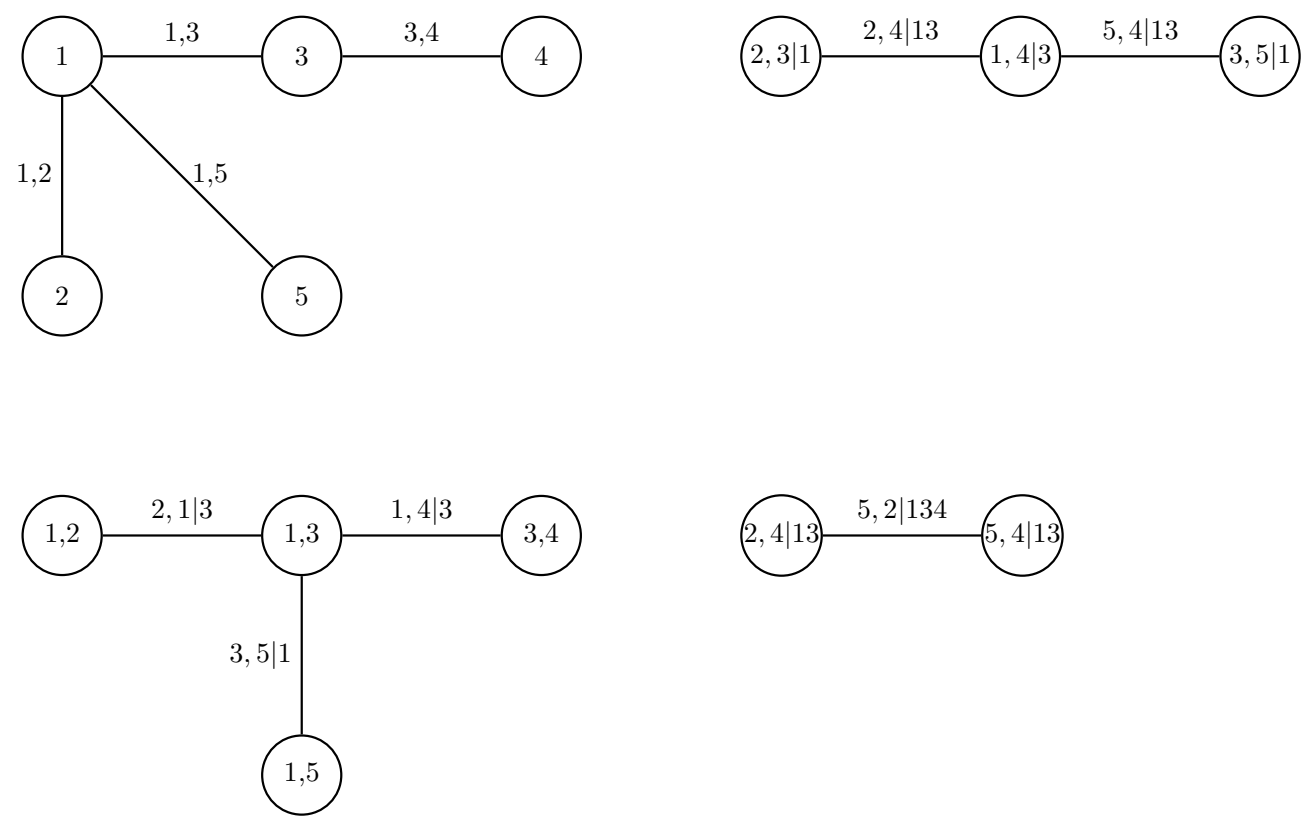

Fig. 3. Graphical illustration of a four-dimensional copula as a regular vine.

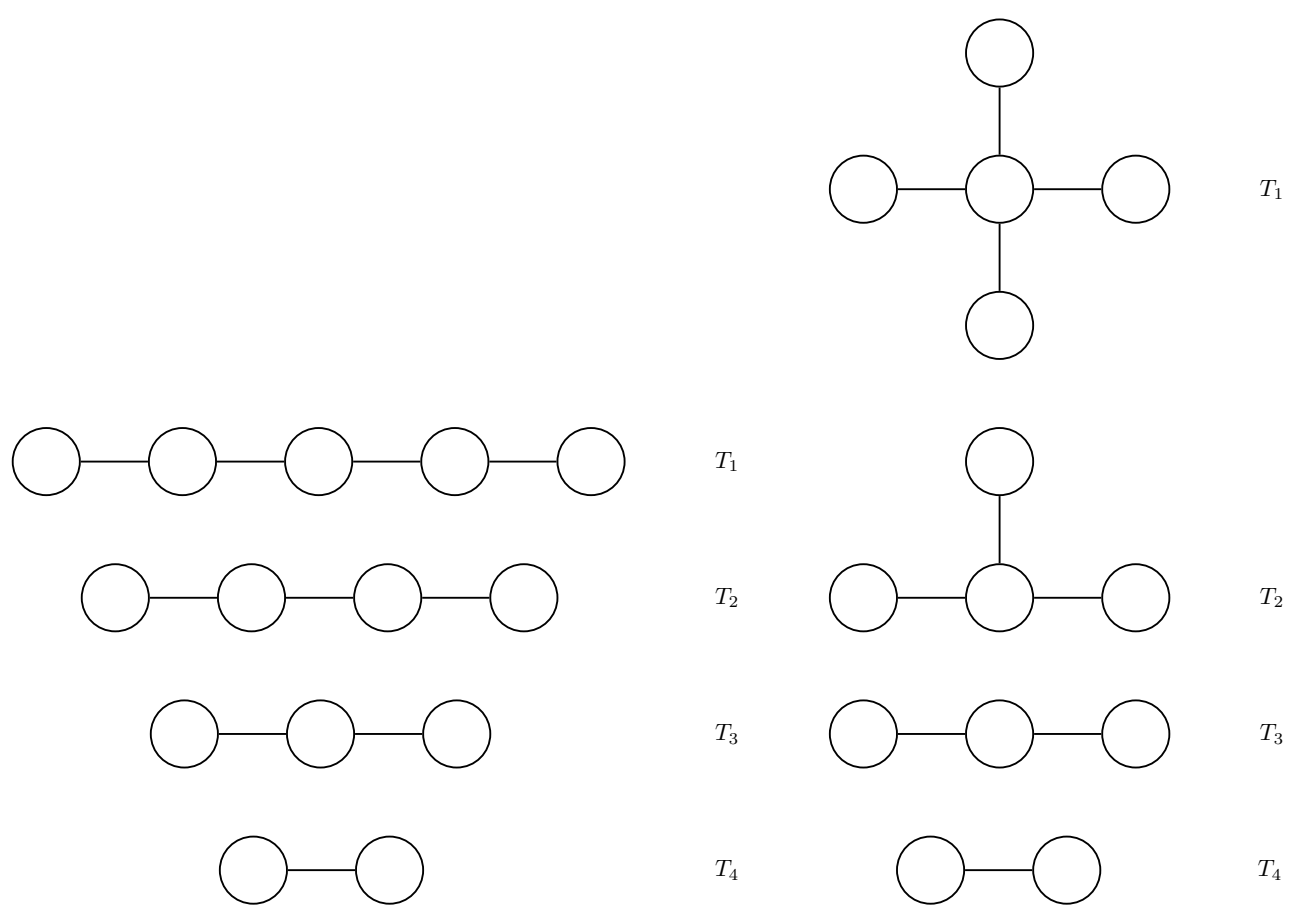

Fig. 4. C-Vine (left) and D-Vine (right) in five dimensions. 


\section{MODELLING DEPENDENCIES}

Recalling from the introduction, the goal of this paper is to model complex dependencies between components of one system as well as interdependencies between components of different systems using copulas. For that reason, the previous section introduced some of the most popular copula families and different methods of constructing high dimensional copulas. This section deals with selecting appropriate copula families for different kinds of failures and investigating the usefulness of the copula construction methods in regards to the reliability analysis of complex networks.

Ideally, the dependency structures and therefore the copulas should be inferred from the measured component failure times of the dependent networks. However, as this data is rarely available for complex systems and the aim of this work is to prove the suitability of copulas in this framework, this is left for future work. Instead, a qualitative approach to the modelling of different kinds of failures is chosen. Examples of how to model two distinct classes of failures are given in the subsequent sections. These qualitative estimates could potentially serve as a basis for deducing the copula structure by Bayesian inference.

\section{Common Cause of Failure}

Common cause of failure is the event that two or more components fail simultaneously due to shared defects [21]. These weaknesses can include but are not limited to [22]:

- Manufacturing defects

- Errors by the maintenance or operator personal

- Shared environmental conditions

This work concentrates on the first weakness, manufacturing defects, especially those manifesting in early component life. The Clayton copula can be used to describe dependence between marginals where there is strong lower tail dependence. Lower (or upper) tail dependence is concept expressing higher dependence in the lower-left (upper-right) quadrant of $[0,1]^{2}$. This property of the Clayton copula is clearly evident from Fig. $1 \mathrm{~b}$ where the samples in the lower-left quadrant are grouped closer together.

Consider a very simple system of two parallel components. The component failure times are assumed to be exponentially distributed with $\lambda=1.5$ and are sampled from a bivariate Clayton copula with $\theta$ chosen such that Kendall's tau equals 0.3. Figure 5 shows a plot of the resulting reliability against the reliability in the independent case. The plot clearly shows how the lower tail dependence translates to the reliability of the system. Initially, the reliability is significantly reduced compared to the independent case. At later points in time, as the dependence weakens, this difference decreases. Contrarily, a copula exhibiting strong upper tail dependence might be used to model common cause failures at high component age.

\section{Interdependencies}

The treatment of interdependencies is not as simple as for common cause of failures. To understand the difficulties it is important to understand the two meanings dependence has in this case. When working with copulas, dependence is a measure of correlation 


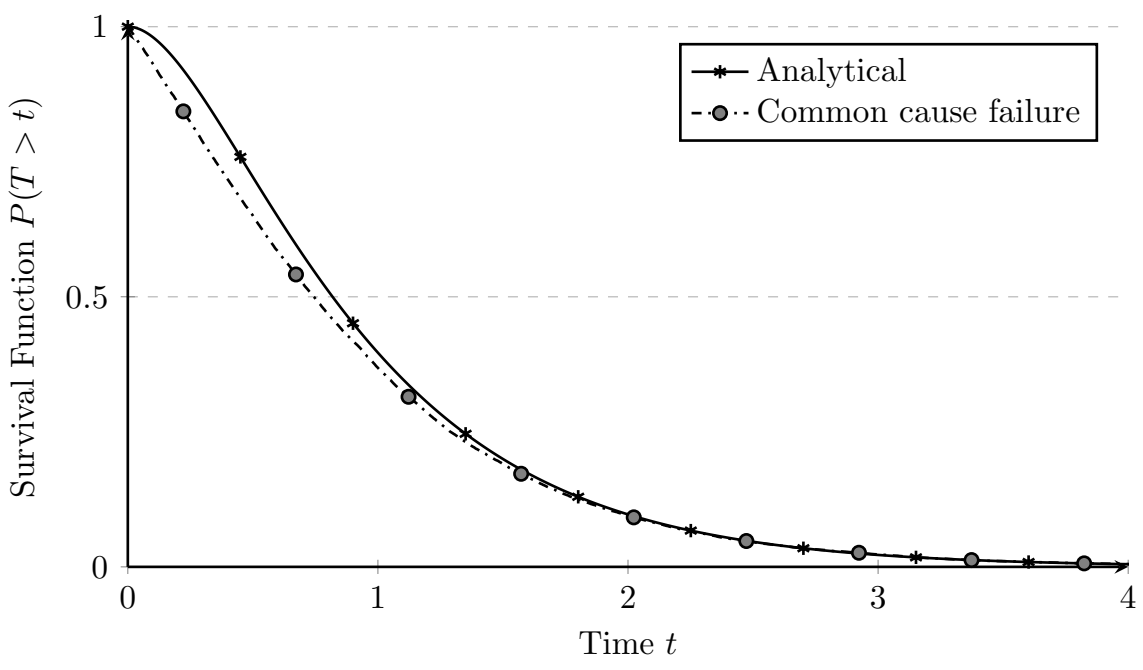

Fig. 5. Reliability of a parallel system subject to common cause of failure.

or concordance and as is the nature of copulas, dependence is modelled independently of the marginals. As such, dependence in a statistical sense does not imply causality. However, this is exactly what interdependencies represent. If one component fails there is a chance that a dependent component will fail as well.

Consider two dependent components whose failure times are distributed with marginal distributions $F_{1}$ and $F_{2}$ and copula $C$, where $F_{1} \neq F_{2}$. If failure times are sampled for both components from a fully dependent copula and apply the marginals using the inverse transformation method, the failure times for the first component will still be distributed according to $F_{1}$ and the failures times for the second component will be distributed with $F_{2}$. Even though perfect dependence is assumed, the components will not fail together. Since the copula approach separates the modelling of the dependence structure from modelling of the marginals, this causality can be included in the latter. In this case, a simple aggregation of the marginals is performed using the resulting strength of dependence (Kendall's tau) as a factor as shown in Eq. 11

$$
U_{1}=(1-\tau) \cdot F_{1}^{-1}\left(u_{1}\right)+\tau \cdot F_{2}^{-1}\left(u_{1}\right)
$$

\section{Construction of the dependence structure}

After selecting appropriate copula families to model the desired failure modes, the overall dependence structure for the network has to be selected. Three approaches exist based on the methods introduced in the previous section. The most straightforward approach is the application of multiple independent copulas to define dependence among groups of components. However, this does not allow for components to be connected to multiple other components by different copula families as one random variable can not be part of two independent copulas and as such is only suitable for simple scenarios. The two more advanced techniques for constructing high dimensional copulas presented are hierarchical Archimedean copulas and vine copulas. While HAC's offer more flexibility in terms of modelling the dependencies, they are still far more restrictive than vine copulas. This is largely due to their nested structure as compared to the graph based 
nature of vine copulas. Additionally, building a graph based dependence structure has obvious synergies with the reliability analysis of networks. For this reason, vine copulas are selected to build the overall dependence structure.

\section{RELIABILITY ANALYSIS}

This section recaps the numerical methodology used to compute the network reliability first introduced in [5]. It is based on the survival signature, an extension of the system signature, and Monte Carlo simulation.

\section{Survival Signature}

The survival signature is a novel tool for the quantification of system and network reliability based on the system signature $[6,23]$. Both signatures allow for a separation of the system structure from its probabilistic characteristics such as component failure times. However, the system signature has a severe limitation in that it is only defined for systems made up of a single component type, which does not apply to complex networks. The survival signature addresses this drawback by generalizing the signature to systems with an arbitrary number of component types.

Consider a system with $m$ components. The state vector is defined as $\underline{x}=\left(x_{1}, \ldots, x_{m}\right)$, where $x_{i}=1$ indicates a component in working condition, while $x_{i}=0$ indicates a component in a failed state. As such, the state vector represents the state of the individual components. The state of the full system is obtained by applying the structure function $\varphi(\underline{x})$ to the state vector. As before, $\varphi(\underline{x})=1$ indicates a working system and $\varphi(\underline{x})=0$ indicates that the system has failed. The structure function is defined based on the problem at hand. In this work, the structure function is assumed to return 1 if a path from any start node to any end node exists for the current network state. Calculating the survival signature for $l$ out of $m$ components working then becomes a combinatorial problem defined as

$$
\Phi(l)=\left(\begin{array}{c}
m \\
l
\end{array}\right)^{-1} \sum_{\underline{x} \in S_{l}} \varphi(\underline{x})
$$

The survival signature is easily extended to systems with multiple component types. Consider a system with $K$ component types, $m_{k}$ components per type $k(k=1, \ldots, K)$ and $l_{k}$ out of $m_{k}$ components per type in a working state, the survival signature becomes

$$
\Phi\left(l_{1}, \ldots, l_{K}\right)=\left[\prod_{k=1}^{K}\left(\begin{array}{c}
m_{k} \\
l_{k}
\end{array}\right)^{-1}\right] \times \sum_{\underline{x} \in S_{l_{1}, \ldots, l_{K}}} \varphi(\underline{x})
$$

As an example, consider a system with two component types and three components per type as illustrated in Fig. 6. Here, node 1 is selected to be the start node and nodes 5 and 6 represent the end nodes. The full survival signature for the network is show in Table 2.

While algorithms to calculate the survival signature have already been available for a number of years [24, 25], efficient computation of the signature for systems with large numbers of components and types still poses a numerical challenge. A new approach attempting to reduce the high computational demand of the survival signature using graph theory and Monte Carlo approximation can be found in [26]. 


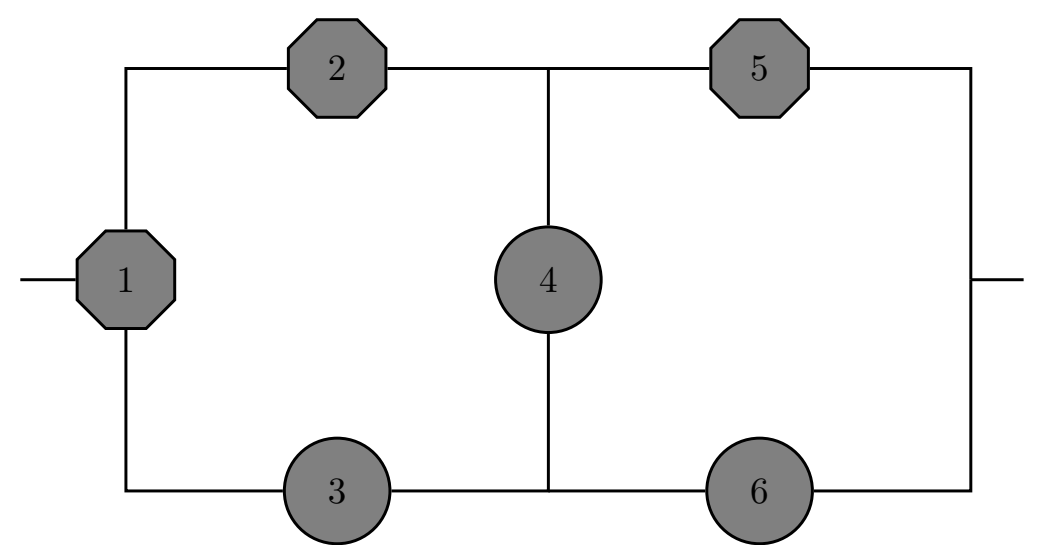

Fig. 6. Network with six components equally divided into two component types.

Table 2. Survival signature of the network shown in Fig. 6.

\begin{tabular}{ccc|ccc}
\hline \hline$l_{1}$ & $l_{2}$ & $\Phi\left(l_{1}, l_{2}\right)$ & $l_{1}$ & $l_{2}$ & $\Phi\left(l_{1}, l_{2}\right)$ \\
\hline \hline 0 & 0 & 0 & 2 & 0 & 0 \\
0 & 0 & 0 & 2 & 1 & 0 \\
0 & 0 & 0 & 2 & 2 & $4 / 9$ \\
0 & 0 & 0 & 2 & 3 & $6 / 9$ \\
1 & 0 & 0 & 3 & 0 & 1 \\
1 & 1 & 0 & 3 & 1 & 1 \\
1 & 2 & $1 / 9$ & 3 & 2 & 1 \\
1 & 3 & $3 / 9$ & 3 & 3 & 1 \\
\hline \hline
\end{tabular}

\section{Survival Function}

Based on the survival signature, the survival function is defined as

$$
P\left(T_{s}>t\right)=\sum_{l_{1}=0}^{m_{1}} \ldots \sum_{l_{k}=0}^{m_{k}} \Phi\left(l_{1}, \ldots, l_{K}\right) P\left(\bigcap_{k=1}^{K}\left\{C_{t}^{k}=l_{k}\right\}\right)
$$

This function gives the probability that a network is still working at time $t$, in other words the reliability of the system. The equation clearly shows the separation of structural information (survival signature on the left) and probabilistic information about component failures (right). This is beneficial as it allows to analyze the network once ahead of the reliability analysis instead of having to re-evaluate the structure every step of the way as with traditional techniques such as fault tree analysis. Additionally, this makes it possible to efficiently run multiple failure scenarios against a network.

\section{Simulation}

Component failure times are sampled from the vine copula, after selecting the number of desired samples $N_{m c}$ and a sufficiently small time step, and transformed 
to their respective marginals. Next, for all combinations $l_{1}, \ldots, l_{K}$ from the survival signature and all time steps $t$ the number of samples representing the exact same combination (amount of components still working at time $t$ ) are counted as $N_{l_{1}, \ldots, l_{K}}(t)$. Then, the probabilistic part of the survival function is approximated by

$$
P\left(\bigcap_{k=1}^{K}\left\{C_{t}^{k}=l_{k}\right\}\right)=\frac{N_{l_{1}, \ldots, l_{K}}(t)}{N_{m c}}
$$

In a final step, the partial reliabilities for all combinations are multiplied by their probability $\Phi\left(l_{1}, \ldots, l_{K}\right)$, introducing the structural information into the reliability, and then summed yielding the full reliability of the network. This means that no computations must be performed for combinations where the probability in the survival signature is zero, further increasing the efficiency of the simulation. This fact is especially useful in higher dimensions where large parts of the survival signature are negligible. A pseudo-algorithm illustrating how to obtain the survival function of the system based on the survival signature and the failure times sampled from the vine copula is given in Algorithm 1. The analytically and numerically computed survival functions for the network shown in Fig. 6 assuming independent exponential failure distributions for the components with $\lambda_{1}=0.8$ and $\lambda_{2}=1.6$ are presented in Fig. 7 .

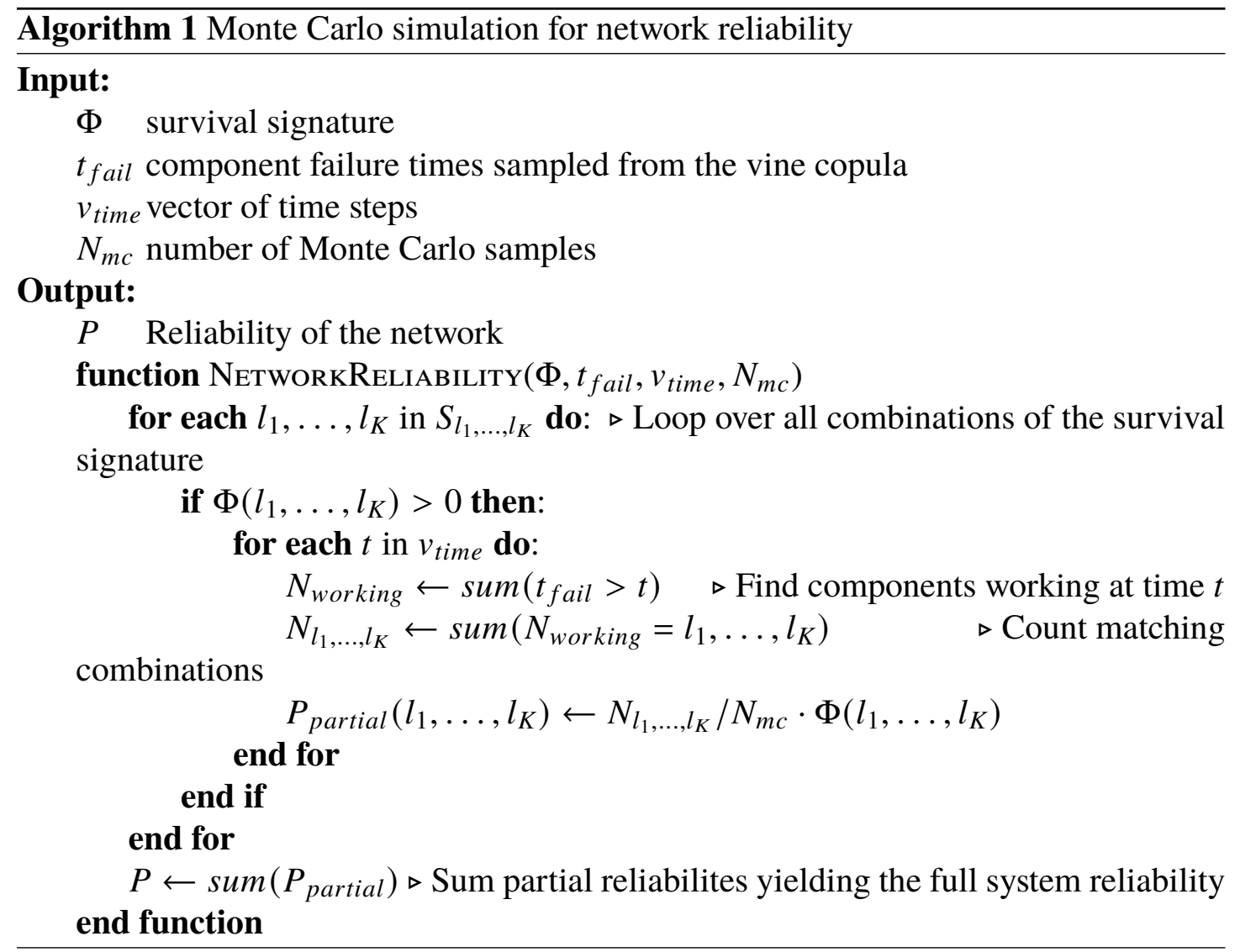




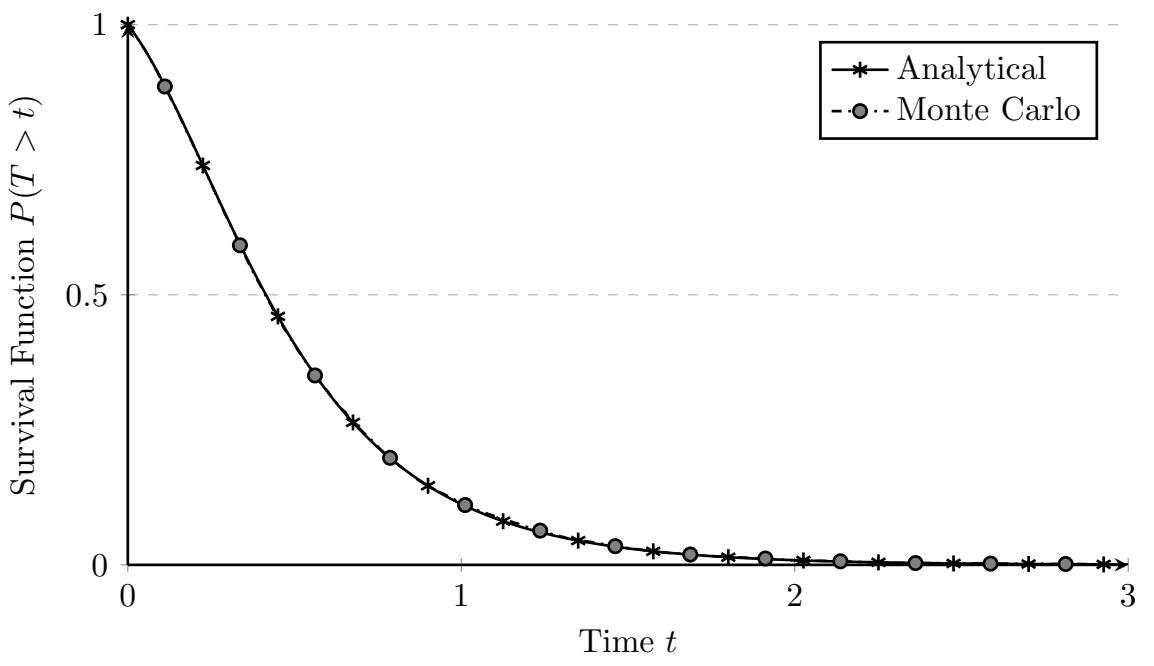

Fig. 7. Survival function for the network in Fig. 6.

\section{Imprecise Reliability Analysis}

Two types of uncertainties must be considered during the reliability analysis, namely, aleatory and epistemic uncertainties. Aleatory uncertainty describes the natural randomness inherent in a process such as component degradation and external forces affecting the system (natural hazards, earthquakes, etc.), while epistemic uncertainty represents the uncertainty due to vagueness in information or a lack thereof. The latter is usually regarded as reducible through acquiring of additional data and information.

Aleatory uncertainty is naturally handled by the reliability analysis technique. Through assuming failure time distributions for the component failures and sampling these during Monte Carlo simulation, the randomness that the model is subject to is included in the analysis. However, the selection appropriate failure time distributions is typically based on either data or expert knowledge, neither of which yields perfect results. In turn, this introduces epistemic uncertainty into the model. These epistemic uncertainties can be tackled by using imprecise probability methods where instead of a single model, a set of plausible models is applied. As a result, the uncertainty propagates through the models and is ultimately reflected in the probability of failure, i.e. the reliability of the network.

Consider two non-decreasing functions $\underline{F}$ and $\bar{F}$ mapping the real line $\mathbb{R}$ into $[0,1]$, with $\underline{F}(x) \leq \bar{F}(x)$ for all $x \in \mathbb{R}$. Let $[\bar{F}(\bar{x}), \underline{F}(x)]$ denote the set of non-decreasing functions mapping $\mathbb{R}$ into $[0,1]$ such that $\underline{F}(x) \leq F(x) \leq \bar{F}(x)$. When $\bar{F}$ and $\underline{F}$ circumscribe an imprecisely known probability distribution, $[\bar{F}, \underline{F}]$ is called the probability box or $p$-box of said probability distribution. As a result, if $[\bar{F}, \underline{F}]$ is a p-box for a random variable $X$ whose distribution is known to be within the p-box, then $\bar{F}(x)$ and $\underline{F}(x)$ are the upper and lower bounds on $F(x)$, respectively [27]. An example of an exponential p-box with parameters $\lambda \in[1.2,2.2]$ is shown in Fig. 8. In this case, only two CDFs need to be computed to fully define the p-box. However, for most families of distributions, four or more CDFs must be evaluated [27]. 


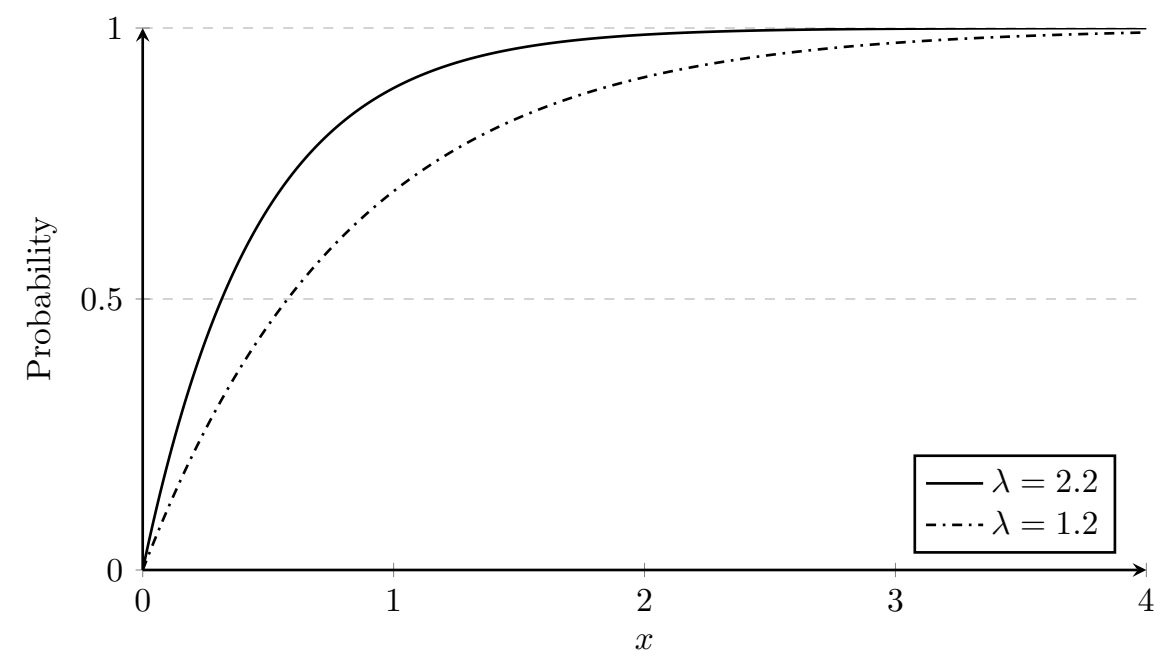

Fig. 8. Example of an exponential p-box with $\lambda \in[1.2,2.2]$.

By feeding the bounds of the p-box into the reliability analysis, the epistemic uncertainty propagates into the result. Thus, instead of one survival function, the result is an upper and a lower bound [28]. Figure 9a shows an example of the upper and lower bounds obtained by performing a reliability analysis of a simple system of two parallel components of the same type, assuming the p-box shown in Fig. 8 for the failure time distributions.

Similarly to a p-box, instead of considering just one precise copula, a set of copulas can be considered to account for uncertainty in the dependencies. Pelessoni, Vicig, Montes, and Miranda [29] generalized p-boxes to the bivariate case. If $\underline{C}$ and $\bar{C}$ are two copulas such that $\underline{C} \leq \bar{C}$, then $[\underline{C}, \bar{C}]$ forms an imprecise copula. Let $\left[\underline{F}_{X}, \bar{F}_{X}\right]$ and $\left[\underline{F}_{Y}, \bar{F}_{Y}\right]$ be two univariate p-boxes for the random variables $X$ and $Y$ respectively, then $[\underline{F}, \bar{F}]$ defined by

$$
\underline{F}(x, y)=\underline{C}\left(\underline{F}_{X}(x), \underline{F}_{Y}(y)\right)
$$

and

$$
\bar{F}(x, y)=\bar{C}\left(\bar{F}_{X}(x), \bar{F}_{Y}(y)\right)
$$

is a bivariate p-box [30]. As with the p-box, defining an imprecise copula from an interval on it's parameters imposes bounds on the system reliability. Consider again a simple system of two parallel components, in this case interlinked by an imprecise Gaussian copula with $\rho \in[0.3,0.6]$. The upper and lower bounds for the reliability are presented in Fig. 9b.

\section{NUMERICAL EXAMPLE}

The network structures for the following numerical example are taken from the IEEE Reliability Test System (RTS) [31]. The system is effectively split into two sub-systems (see Fig. 10 and Fig. 11) by removing the the transformers that link the low power to the high power grid. Components in the networks are classified into five types. Component types 1 and 5 are the non-generating nodes in networks 1 and 2 respectively. The 


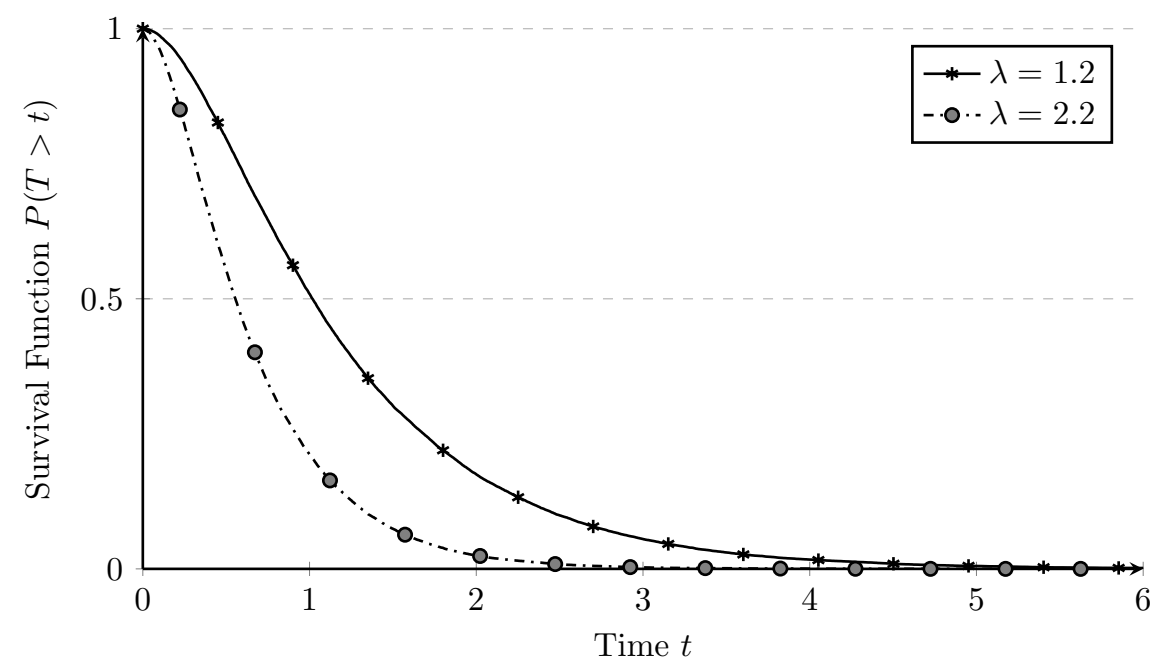

(a) Probability-box

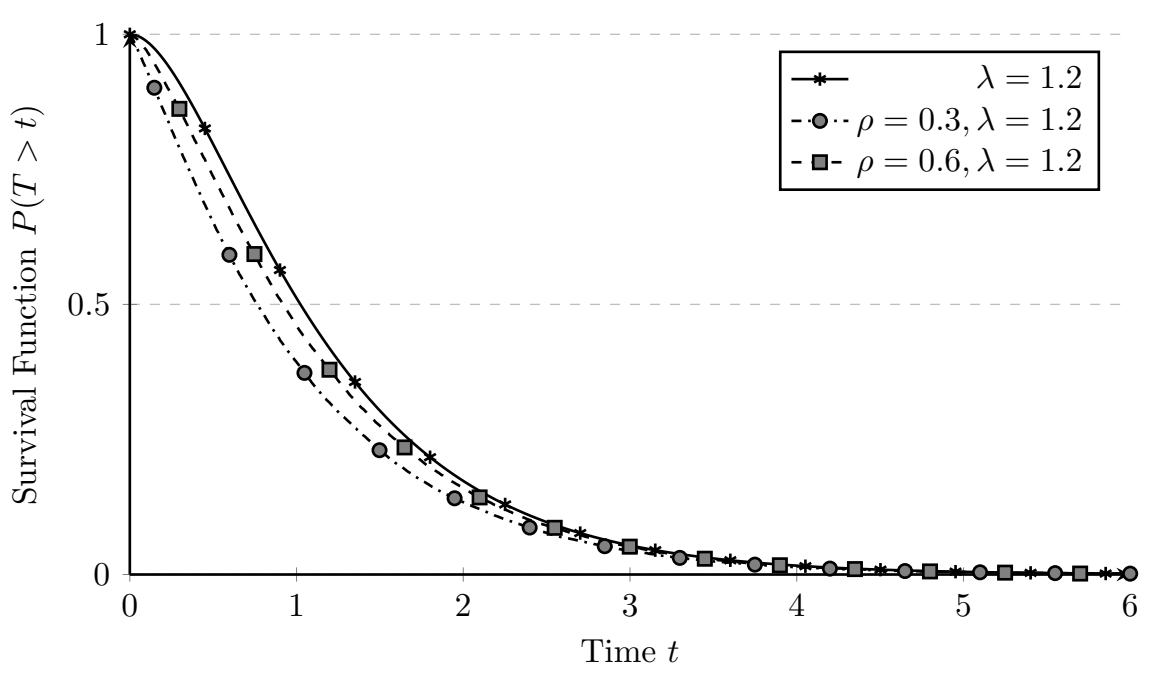

(b) Imprecise copula

Fig. 9. Bounds on the reliability resulting from applying a p-box (a) or an imprecise Gaussian copula (b) to a simple system of two parallel components.

generating nodes are divided into three component types 2, 3 and 4. These represent different types of generators such as nuclear, oil or coal power plants. Note that this is no attempt at solving the IEEE RTS. The system is merely providing the network topology.

In a first step to obtain the reliability, the required survival signatures for both networks are calculated using the approach presented in [26]. Next, the vine copula that is used for sampling the individual component failure times is assembled from bivariate copulas. A common cause of failure is set among the groups of nodes of types 2, 3, and 4 through imprecise bivariate Clayton copulas. Next, the transformers that were removed to split the network in two, are reintroduced as interdependencies between 


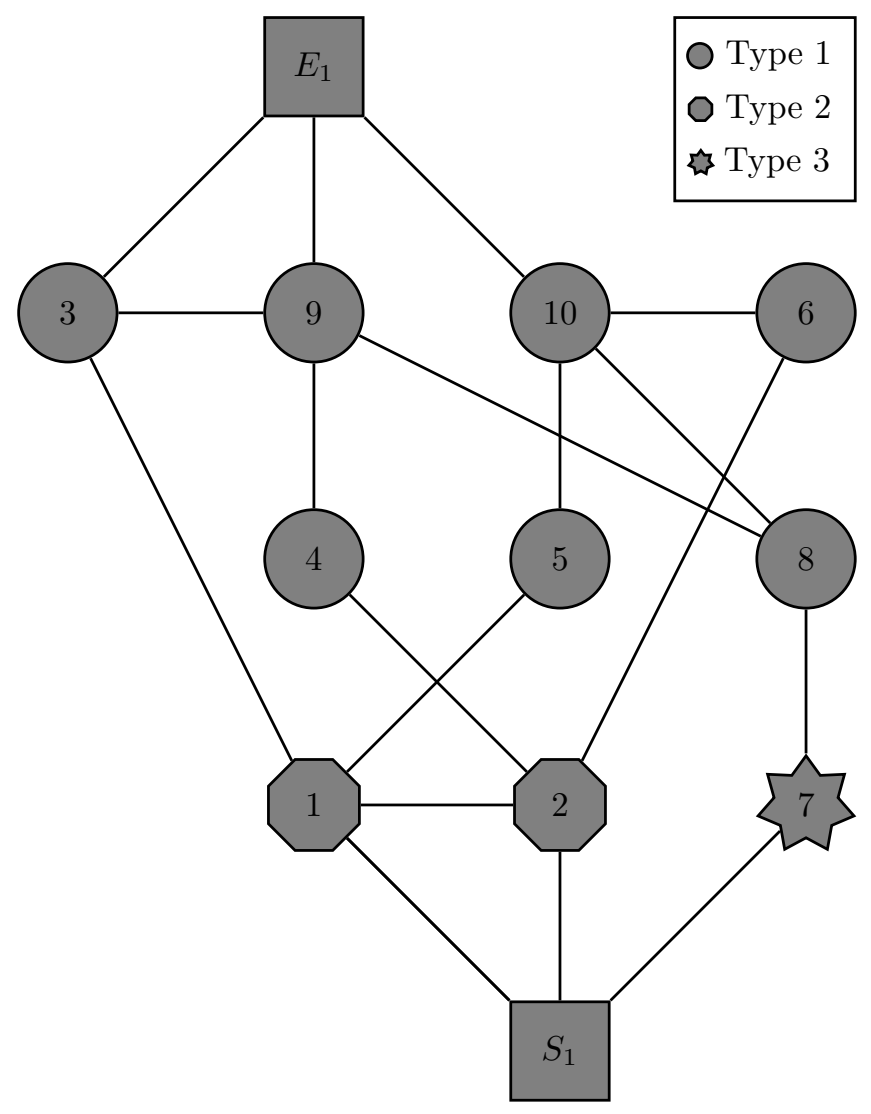

Fig. 10. Structure of the first network taken from the IEEE RTS

the nodes 3 and 11, 9 and 24 as well as 10 and 12 using imprecise bivariate Gaussian copulas. All one-dimensional marginal distributions are assumed to be exponentially distributed. The parameters for the marginals and the copulas are presented in Tab. 3.

Finally, the reliability analysis is performed using the previously introduced Monte Carlo simulation method. The upper and lower bounds of the reliability for network 1 is presented in Fig.12. For comparison, the plot also contains a deterministic reliability analysis (all mean values) of network 1.

\section{CONCLUSION}

This paper presented a novel approach to the modelling of complex dependencies in interdependent networks by leveraging multivariate copulas. Over the course of this work the necessary theory on copulas, dependence measures and pair copula construction techniques was discussed. Of the investigated structures vine copulas have shown to be ideally suited to model higher dimensional dependencies with sufficient flexibility. The capabilities of the proposed approach were highlighted using a scenario based on the network topology of the IEEE Reliability Test System. The application of vine copulas has proven to be able to represent a complicated model with multiple competing failure modes. It was shown that imprecision can easily be included in the reliability analysis. Nonetheless, the modelling flexibility of this method comes at a price. Finding a suitable vine copula structure is not a trivial task and greatly suffers 


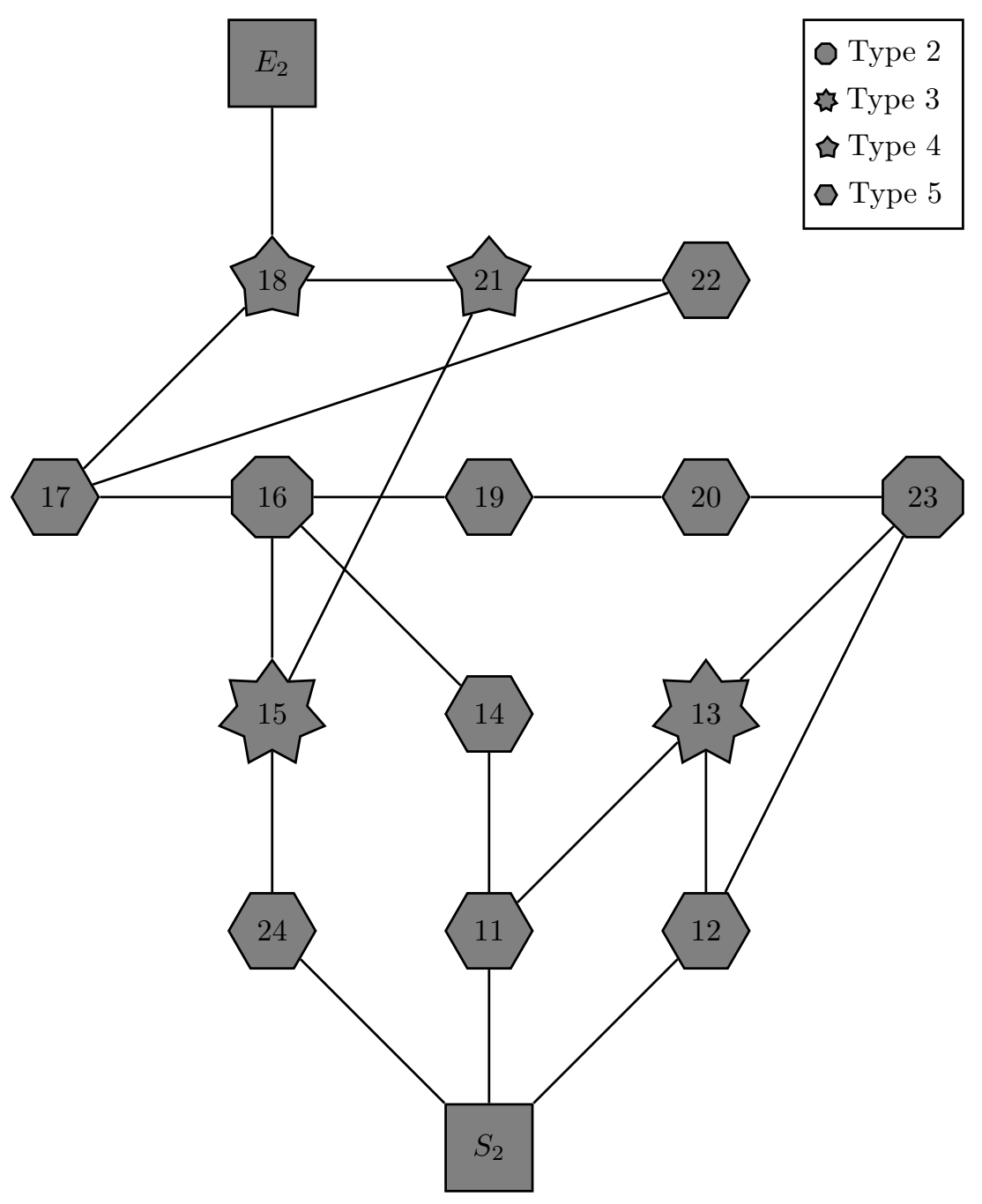

Fig. 11. Structure of the second network taken from the IEEE RTS

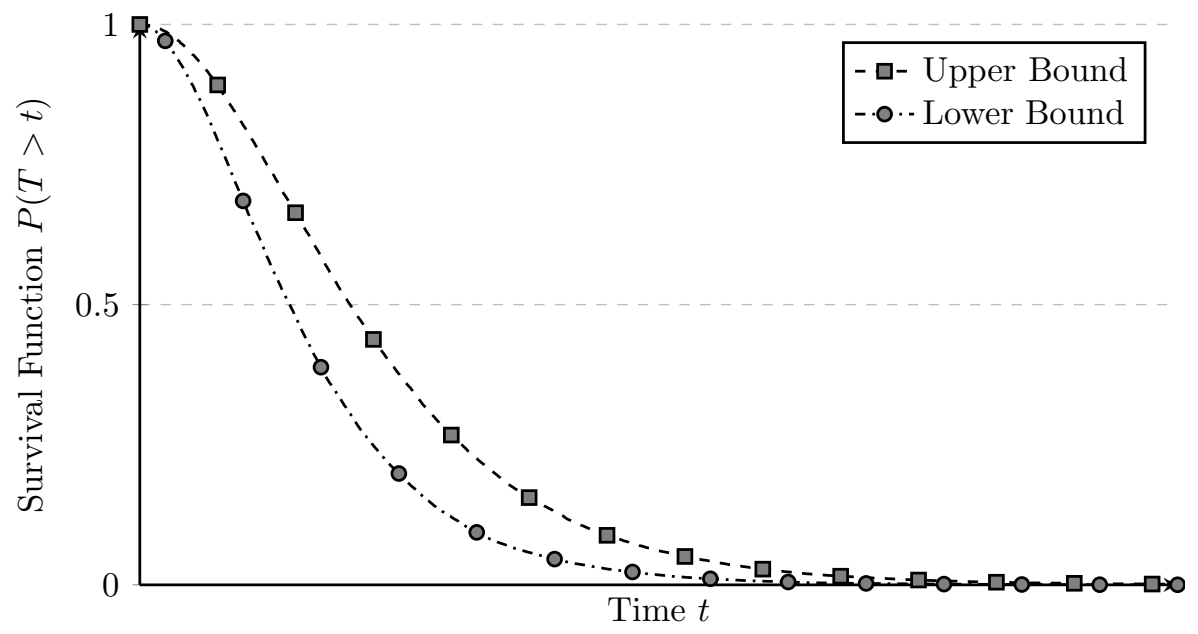

Fig. 12. Bounds on the reliability of network 1 . 
Table 3. Failure rate ranges of the exponential marginal distributions and copula parameters used in the numerical example.

\begin{tabular}{llc}
\hline \hline Parameter & Definition & Parameter range \\
\hline \hline$\lambda_{1}$ & Failure rate of component type 1 & $\lambda_{1} \in[0.8,1.2]$ \\
$\lambda_{2}$ & Failure rate of component type 2 & $\lambda_{2} \in[1.4,1.5]$ \\
$\lambda_{3}$ & Failure rate of component type 3 & $\lambda_{3} \in[1.6,1.9]$ \\
$\lambda_{4}$ & Failure rate of component type 4 & $\lambda_{4} \in[2.0,2.3]$ \\
$\lambda_{5}$ & Failure rate of component type 5 & $\lambda_{5} \in[1.8,2.2]$ \\
$\tau_{1}$ & Clayton copula parameters on component type 2 & $\tau_{1} \in[0.1,0.3]$ \\
$\tau_{2}$ & Clayton copula parameters on component type 3 & $\tau_{2} \in[0.2,0.4]$ \\
$\tau_{3}$ & Clayton copula parameters on component type 4 & $\tau_{3} \in[0.1,0.3]$ \\
$\tau_{4}$ & Gaussian copula parameters between network 1 and 2 & $\tau_{4} \in[0.4,0.8]$ \\
\hline \hline
\end{tabular}

from the curse of dimensionality.

In order to facilitate a transparent illustration of the approach, we have chosen a relatively small system which still carries the key features of a realistic system. The expansion to a large complex system would require a number of additional numerical challenges to be solved. This is beyond the scope of this paper and therefore left for future work. The challenges include deriving the vine copula model from data. Gruber and Czado [32] presented a promising method for Bayesian model selection and inference of vine copulas. The technique is the first to be able to jointly select the vine tree structure and the copula families. Additionally, the modelling aspects of other competing failure events such as external threats (e.g. earthquakes, tsunamis, terrorist attacks) must be closely investigated.

\section{ACKNOWLEDGEMENTS}

This project is partially supported by the European Union's Horizon 2020 Research and Innovation Programme RISE under grant agreement no. 730888 (RESET).

\section{References}

[1] UN-OCHA. Phillipines: Typhoon Hayan Situation Report No. 19. Aug. 2013. URL: http : / / reliefweb . int / report / philippines / philippines typhoon-haiyan-situation-report-no-19-28-november-2013.

[2] Sergey V Buldyrev, Roni Parshani, Gerald Paul, H Eugene Stanley, and Shlomo Havlin. "Catastrophic cascade of failures in interdependent networks". In: Nature 464 (2010), pp. 1025-1028.

[3] William M. Leavitt and John J. Kiefer. "Infrastructure Interdependency and the Creation of a Normal Disaster: The Case of Hurricane Katrina and the City of New Orleans". In: Public Works Management \& Policy 10.4 (2006), pp. 306-314. DOI: $10.1177 / 1087724$ X06289055. 
[4] Louise K. Comfort and Thomas W. Haase. "Communication, Coherence, and Collective Action: The Impact of Hurricane Katrina on Communications Infrastructure". In: Public Works Management \& Policy 10.4 (2006), pp. 328-343. DoI: 10.1177/1087724X06289052.

[5] Jasper Behrensdorf, Sebastian Brandt, Matteo Broggi, and Michael Beer. "Numerically efficient reliability analysis of complex interdependent networks". In: Safety and Reliability. Theory and Applications. Ed. by Marko Cepin and Radim Bris. CRC Press, 2017.

[6] Frank P. A. Coolen and T. Coolen-Maturi. "Generalizing the signature to systems with multiple types of components". In: Complex Systems and Dependability. Ed. by W. Zamojski, J. Mazurkiewicz, J. Sugier, T. Walkowiak, and J. Kacprzyk. Berlin: Springer, 2012, pp. 115-130.

[7] E. Patelli, G. Feng, F. P. A. Coolen, and T. Coolen-Maturi. "Simulation methods for system reliability using the survival signature". In: Reliability Engineering \& System Safety 167 (2017), pp. 327-337.

[8] Doris Schirmacher and Ernesto Schirmacher. Multivariate Dependence Modeling using Pair-Copulas. Technical Report. Chicago, IL: Society of Actuaries: 2008 Enterprise Risk Management Symposium, Apr. 2008.

[9] Umberto Cherubini, Elisa Luciano, and Walter Vecchiato. Copula methods in finance. John Wiley \& Sons, 2004.

[10] Barry K Goodwin and Ashley Hungerford. "Copula-based models of systemic risk in US agriculture: implications for crop insurance and reinsurance contracts". In: American Journal of Agricultural Economics 97.3 (2014), pp. 879-896.

[11] LSVP Zhang and VP Singh. "Bivariate flood frequency analysis using the copula method". In: Journal of hydrologic engineering 11.2 (2006), pp. 150-164.

[12] Harry Joe. Dependence modeling with copulas. Vol. 134. Monographs on Statistics \& Applied Probability. Chapman and Hall/CRC, 2014.

[13] Jun Yan. "Multivariate modeling with copulas and engineering applications". In: Springer Handbook of Engineering Statistics. Springer, 2006, pp. 973-990.

[14] Mangey Ram and SB Singh. "Analysis of reliability characteristics of a complex engineering system under copula". In: Journal of Reliability and Statistical Studies 2.1 (2009), pp. 91-102.

[15] Michael Beer, Scott Ferson, and Vladik Kreinovich. "Imprecise probabilities in engineering analyses". In: Mechanical systems and signal processing 37.1-2 (2013), pp. 4-29.

[16] R. B. Nelson. An Introduction to Copulas. second. Springer Series in Statistics. New York: Springer Science \& Business Media, 2007.

[17] M Sklar. "Fonctions de repartition an dimensions et leurs marges". In: Publ. Inst. Statist. Univ. Paris 8 (1959), pp. 229-231. 
[18] Jan-Frederik Mai and Matthias Scherer. Simulating copulas: stochastic models, sampling algorithms, and applications. Vol. 4. Series in Quantative Finance. London: Imperial College Press, 2012.

[19] Marius Hofert and Martin Mächler. "Nested Archimedean copulas meet R: The nacopula package”. In: Journal of Statistical Software 39.9 (2011), pp. 1-20.

[20] Malte Kurz. VineCopulaMatlab toolbox. July 2016. urL: https: / / gi thub. com/MalteKurz/VineCopulaMatlab.

[21] I. A. Watson. Review of common cause failures. UKAEA, 1981.

[22] B. J. Hanks. "An appreciation of common cause failures in reliability". In: Proceedings of the Institution of Mechanical Engineers, Part E: Journal of Process Mechanical Engineering 212.1 (1998), pp. 31-35.

[23] Francisco J Samaniego. System signatures and their applications in engineering reliability. Vol. 110. International Series in Operations Research \& Management Science. Springer US, 2007.

[24] L. J. M. Aslett. ReliabilityTheory: Tools for structural reliability analysis. Aug. 2012. URL: http: //WWW. louisaslett. com.

[25] S. Reed. "An efficient algorithm for exact computation of system and survival signatures using binary decision diagrams". In: Reliab Eng Syst Safe 165 (Sept. 2017), pp. 257-267.

[26] Jasper Behrensdorf, Sebastian Brandt, Matteo Broggi, and Michael Beer. "Efficient Approximation of the Survival Signature for Large Networks". In: Proceedings of the 6th International Symposium on Reliability Engineering and Risk Management. Ed. by Xudong Qian, Sze Dai Pang, Ghim Ping Raymond Ong, and Kok-Kwang Phoon. Singapore: Research Publishing, 2018.

[27] Scott Ferson, Vladik Kreinovich, Lev Grinzburg, Davis Myers, and Kari Sentz. Constructing probability boxes and Dempster-Shafer structures. Tech. rep. SAND2015-4166J. Sandia National Lab.(SNL-NM), Albuquerque, NM (United States), 2015.

[28] Geng Feng, Edoardo Patelli, Michael Beer, and F. P. A. Coolen. "Imprecise system reliability and component importance based on survival signature". In: Reliability Engineering \& System Safety 150 (2016), pp. 116-125.

[29] Renato Pelessoni, Paolo Vicig, Ignacio Montes, and Enrique Miranda. "Bivariate p-boxes". In: International Journal of Uncertainty, Fuzziness and KnowledgeBased Systems 24.02 (2016), pp. 229-263.

[30] Ignacio Montes, Enrique Miranda, Renato Pelessoni, and Paolo Vicig. "Sklar's theorem in an imprecise setting”. In: Fuzzy Sets and Systems 278 (2015), pp. 4866. 
[31] Cliff Grigg, Peter Wong, Paul Albrecht, Ron Allan, Murty Bhavaraju, Roy Billinton, Quan Chen, Clement Fong, Suheil Haddad, Sastry Kuruganty, et al. "The IEEE reliability test system-1996. A report prepared by the reliability test system task force of the application of probability methods subcommittee". In: IEEE Transactions on power systems 14.3 (1999), pp. 1010-1020.

[32] Lutz F. Gruber and Claudia Czado. "Bayesian Model Selection of Regular Vine Copulas". In: Bayesian Anal. 13.4 (Dec. 2018), pp. 1111-1135. Dor: 10.1214/ 17-BA1089. 